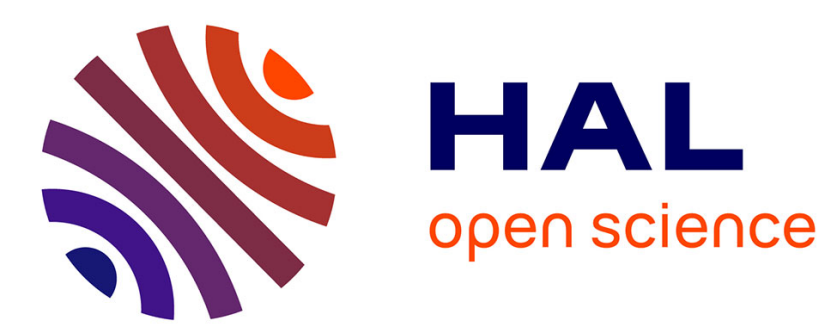

\title{
On the Habitability and Future Exploration of Ocean Worlds
}

\author{
K. Hand, C. Sotin, A. Hayes, A. Coustenis
}

\section{To cite this version:}

K. Hand, C. Sotin, A. Hayes, A. Coustenis. On the Habitability and Future Exploration of Ocean Worlds. Space Science Reviews, 2020, 216 (5), 10.1007/s11214-020-00713-7 . insu-02993658

\section{HAL Id: insu-02993658 https://hal-insu.archives-ouvertes.fr/insu-02993658}

Submitted on 16 Nov 2020

HAL is a multi-disciplinary open access archive for the deposit and dissemination of scientific research documents, whether they are published or not. The documents may come from teaching and research institutions in France or abroad, or from public or private research centers.
L'archive ouverte pluridisciplinaire HAL, est destinée au dépôt et à la diffusion de documents scientifiques de niveau recherche, publiés ou non, émanant des établissements d'enseignement et de recherche français ou étrangers, des laboratoires publics ou privés. 


\title{
On the Habitability and Future Exploration of Ocean Worlds
}

\author{
K.P. Hand ${ }^{1}$ (D) C C. Sotin ${ }^{1}$ - A. Hayes ${ }^{2}$ - A. Coustenis ${ }^{3}$
}

Received: 13 February 2020 / Accepted: 24 June 2020 / Published online: 20 July 2020

(C) This is a U.S. government work and not under copyright protection in the U.S.; foreign copyright protection may apply 2020

\begin{abstract}
Liquid water oceans are now predicted to exist beneath the icy shells of numerous worlds in the outer solar system. These ocean worlds are prime targets in our search for evidence of life beyond Earth, and specifically extant life. Here we review the conditions that may lead to several of these worlds being habitable, and provide a framework for the future exploration of these astrobiologically compelling targets.
\end{abstract}

Keywords Ocean worlds · Astrobiology · Comparative oceanography · Europa · Enceladus · Titan

\section{Introduction}

After half a century of solar system exploration enabled by robotic spacecraft, we now have good evidence for vast, potentially global, liquid water oceans beneath the icy shells of many moons and dwarf planets of the outer solar system (Khurana et al. 1998; Kivelson et al. 2000; Waite et al. 2009; Postberg et al. 2009; Hussmann et al. 2006; Iess et al. 2012; Kivelson et al. 2002; Nimmo et al. 2016; Stern et al. 2018). These oceans provide new frontiers for scientific exploration; specifically, for understanding oceans, and aqueous geochemical processes, as a widespread planetary phenomenon. Furthermore, and perhaps most intriguingly, these ocean worlds provide locales that may have given rise to life, and which could host life into the present epoch.

Ocean Worlds

Edited by Athena Coustenis, Tilman Spohn, Rafael Rodrigo, Kevin P. Hand, Alexander Hayes, Karen Olsson-Francis, Frank Postberg, Christophe Sotin, Gabriel Tobie, Francois Raulin and Nicolas Walter

K.P. Hand

Kevin.P.Hand@jpl.nasa.gov

1 Jet Propulsion Laboratory, 4800 Oak Grove Drive, Pasadena, CA 91109, USA

2 Cornell University, Ithaca, NY, USA

3 LESIA, PSL University, CNRS, Observatoire de Paris-Meudon, 5, pl. Jules Janssen, 92195 Meudon, France 
Searching for extant (living) life in our solar system is critical if we are to truly understand biology as a potentially common phenomenon in our universe. Such a discovery would permit analyses of the organism's fundamental biochemistry and could - depending on locale - indicate a second origin of life, independent from the genetic tree that roots all life on Earth (Lazcano and Hand 2012). Extant life also enables an examination of contingent versus convergent evolutionary solutions to biology and biochemistry. For example, is there an alternative to the DNA, RNA, ATP, and protein paradigm that drives all life on Earth, or is our biochemistry a common solution on which chemistry converges along the path toward the emergence of biology?

The habitability of a world, however, is distinct from whether or not that world actually harbors life; the origin of life may serve as a bottleneck for populating potentially habitable worlds. At present, we lack a well-constrained understanding of detailed conditions that lead to, or prohibit, the origin of life. Our ability to assess and predict which worlds in our solar system gave rise to life is, therefore, limited. Through our study of life on Earth, however, we can measure the conditions under which life as we know it survives, thus providing valuable metric for constraining habitability. Broadly, habitability can be considered to be the conditions required for sustaining life as we know it. Habitability can be predicted, based on extrapolation from well-studied environments on Earth, whereas the origin of life cannot, given the uncertainty on the requirements for origins.

Here we briefly review the primary conditions, or keystones, for habitability and provide an assessment of candidate ocean worlds in this context. Importantly, though the keystones for life may be universal, here we focus on carbon- and water-based life, i.e., life that utilizes water as its primary solvent and carbon as its primary building block. For life that might utilize completely different chemistries, we direct the reader to the comprehensive and insightful review by Benner and colleagues (Benner et al. 2004).

\section{Keystones of Habitability}

The three keystones for life as we know it are: 1) access to liquid water, 2) access to the elements that serve as the building blocks for life, and 3) access to some form of chemical or electromagnetic energy from which the energy needed to power life can be derived (Nealson 1997).

Figure 1, based on (Hand et al. 2009), highlights these keystones and the candidate ocean worlds that may satisfy these criteria. We discuss each of these in more detail in the sections that follow. We caution that this is a notional diagram based on a combination observations and models; new data and improved models could certainly lead to alternative arrangements of these worlds, and the respective keystones. In this Venn diagram all of the candidate contemporary ocean worlds, by definition, fall into the circle for liquid water. Six worlds Ganymede, Callisto, Europa, Enceladus, Titan, and Pluto - lie in the intersection of water and the elements needed for life. Three worlds - Europa, Enceladus, and Titan - lie in the intersection of liquid water, elements, and the energy needed for life. Triton and Mimas, as indicated, may each harbor an ocean (Schenk and Zahnle 2007; Stern and McKinnon 2000; Tajeddine et al. 2014; Hussmann et al. 2006), but too little is known about their surface and subsurface chemistry to warrant the attribution of any additional keystones. Ceres, on the other hand, likely lacks a large ocean in the modern epoch, but aqueous alteration in the past may have led to a subsurface rich with the elements, and possibly the chemical or photolytic energy needed for life (De Sanctis et al. 2017; Combe et al. 2016; Ammannito et al. 2016). See De Sanctis et al. (2020) for more detail on Ceres. Included in the circles of the Venn 
Fig. 1 The keystones for the habitability of life as we know it. Access to liquid water, the elements needed to build life, and some form of energy to power life constitute the three primary keystones. Within each of the keystones are environmental attributes that lead to that keystone being satisfied in a given environment. Within the Venn diagram of these keystones are placed several of the ocean worlds in our solar system. This notional diagram is based on a combination observations and models, and we emphasize that new data and improved models could yield alternative arrangements
Keystones of Habitability

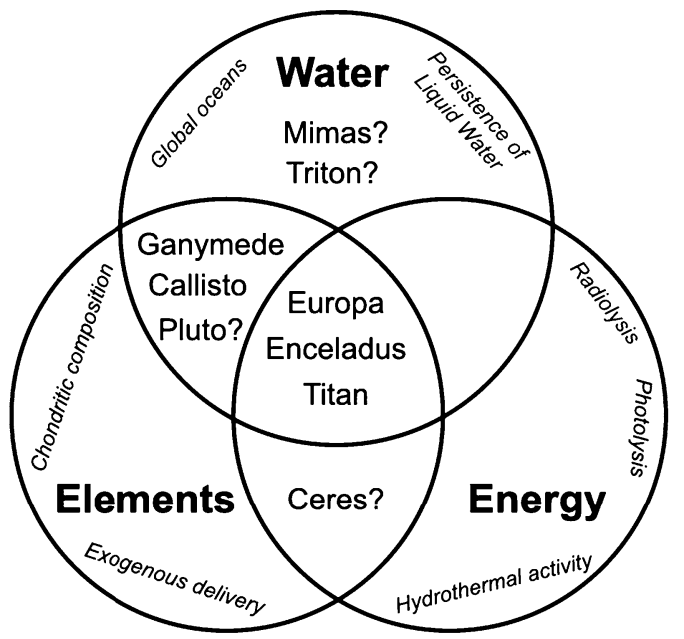

diagram are a few of the processes and parameters associated with each keystone. Time, and the persistence of a stable, habitable environment, could be considered a forth keystone, and we also address this factor in the sections below.

With life on Earth as a guide, liquid water is perhaps the primary keystone for habitability and sustaining the conditions needed for life as we know it. As a result, the search for evidence of life within our solar system has largely been driven by the search for liquid water, past or present, on the surfaces of worlds. NASA's mantra for the search for life beyond Earth has long been 'follow the water', and the ocean worlds harbor vast quantities of liquid water.

To this end, the habitable zone for planets has traditionally been defined, with many variations, by stellar irradiance and the energy needed to sustain regions of liquid water on planetary surfaces (see e.g., (Kasting et al. 1993)). For a given star, the region within which planets can maintain liquid water oceans on their surfaces results in a planetary 'Goldilocks' zone for habitability - too close to the star and surface water boils away, too far away and water freezes. Venus, Earth, and Mars serve as the obvious archetype for this Goldilocks scenario: Venus is too close and too hot for liquid water (in the modern epoch), Mars is too far away and too cold for liquid water on its surface (again, in the modern epoch), but Earth is at just the right distance from our G2V yellow dwarf star so as to sustain liquid water oceans on the surface of our planet (Kuiper 1938; Salpeter 1955; Keenan and McNeil 1989).

The ocean worlds of the outer solar system, however, break away from this framework and provide a new 'Goldilocks' for habitability. Tidal energy and radiogenic decay sustain subsurface oceans beneath the floating ice shells of numerous moons and worlds of the outer solar system.

The availability of liquid water for sustaining a habitable environment is, perhaps by definition, satisfied by merit of being an ocean world. Here we do not review this first keystone in detail, but in Sect. 2.3 we provide a brief assessment of the key observations that have facilitated the discovery of numerous oceans. The detailed evidence for subsurface liquid water has been covered thoroughly elsewhere (Khurana et al. 1998; Kivelson et al. 
2000, 2002; Waite et al. 2009; Postberg et al. 2009; Hussmann et al. 2006; Iess et al. 2012; Nimmo et al. 2016; Stern et al. 2018).

Importantly, however, we note that worlds like Ceres perhaps represent a transitional class of ocean world. Ceres may have had a large habitable subsurface ocean in the past, but may no longer retain enough heat to sustain a substantial, oceanic volume of liquid water (De Sanctis et al. 2017; Combe et al. 2016; Ammannito et al. 2016; Neveu and Desch 2015; Hesse and Castillo-Rogez 2019; Ermakov et al. 2017; Fu et al. 2017). Worlds such as Ceres may offer useful comparisons for the evolution of subsurface aqueous environments over geologic time, and could contain evidence of extinct or extant life, were it to have arisen within such an ocean. In this context, Ceres is perhaps similar to Mars in many ways, but also bears some similarity to the smaller bodies of the outer solar system that harbor subsurface oceans.

Beyond the identification of liquid water oceans, several physical and chemical parameters that characterize aqueous environments are important to consider in the context of habitability. The liquid water temperature, pressure, $\mathrm{pH}$, alkalinity, and salinity predicted for these oceans all fall well within ranges that life on Earth can tolerate. None of these parameters outright appears to be a limiting factor for habitability (Rothschild and Mancinelli 2001), though one or more could impose strong limitations on the origin of life itself.

\subsection{On the Elements Required to Build Life}

The second keystone, the availability of the elements needed to build life, is a more complicated parameter. Life utilizes at least 54 elements from the periodic table, many of which are essential to life (Wackett et al. 2004). By merit of being further out in the solar system than the rocky terrestrial planets, and likely having formed well beyond the ice lines of many volatile species (e.g., $\mathrm{H}_{2} \mathrm{O}, \mathrm{SO}_{2}, \mathrm{CO}_{2}, \mathrm{CH}_{4}$, and $\mathrm{NH}_{3}$ ), the ice covered ocean worlds likely formed with a substantial inventory of the lighter elements that life requires (Fray and Schmitt 2009). On many of these worlds, the volatiles listed above have been observed - spectroscopically or spectrometrically - in the surface ice, or in their atmospheres or exospheres (Coustenis et al. 2010; Johnson 2004). Radiolytic and photolytic processing, coupled with diurnal and seasonal temperature variations, lead to many complex cycles between the vapor and solid phases of these volatiles (Johnson 1990).

Figure 2 shows the distribution of the light, biologically essential elements in the solar system, normalized to solar abundances. This plot, based on (McKay 1991; Hand et al. 2009), illustrates the increased abundance of the light elements in the outer solar system, relative to the inner solar system. The relative ratios of $\mathrm{C}, \mathrm{N}, \mathrm{P}$, and $\mathrm{S}$, provides a general guide for the availability of the essential elements needed for life as we know it. Beyond the asteroid belt, carbon is over two orders of magnitude more abundant than in the inner solar system; nitrogen is approximately four orders of magnitude more abundant, and sulfur is likely enhanced by a factor of a few. Only phosphorous is slightly depleted in the outer solar system. As with Earth, much of the phosphorous is likely sequestered in silicates, such as apatite. The availability of phosphorous within ocean worlds may therefore be a function of water-rock interactions that could occur within the seafloors of these worlds.

We note that the formation, evolution, and resulting composition of each ocean world is distinct from the planetary compositions represented by this diagram, but the broad trend indicates that the outer solar system may have an elemental advantage over the inner solar system when it comes to the availability of carbon, nitrogen, and sulfur.

While the light elements may be the most essential to life, heavier elements, such as iron and magnesium, are also required. The availability of these heavier elements can be assessed in part by considering the bulk densities of the ocean worlds (Hussmann et al. 2006; 


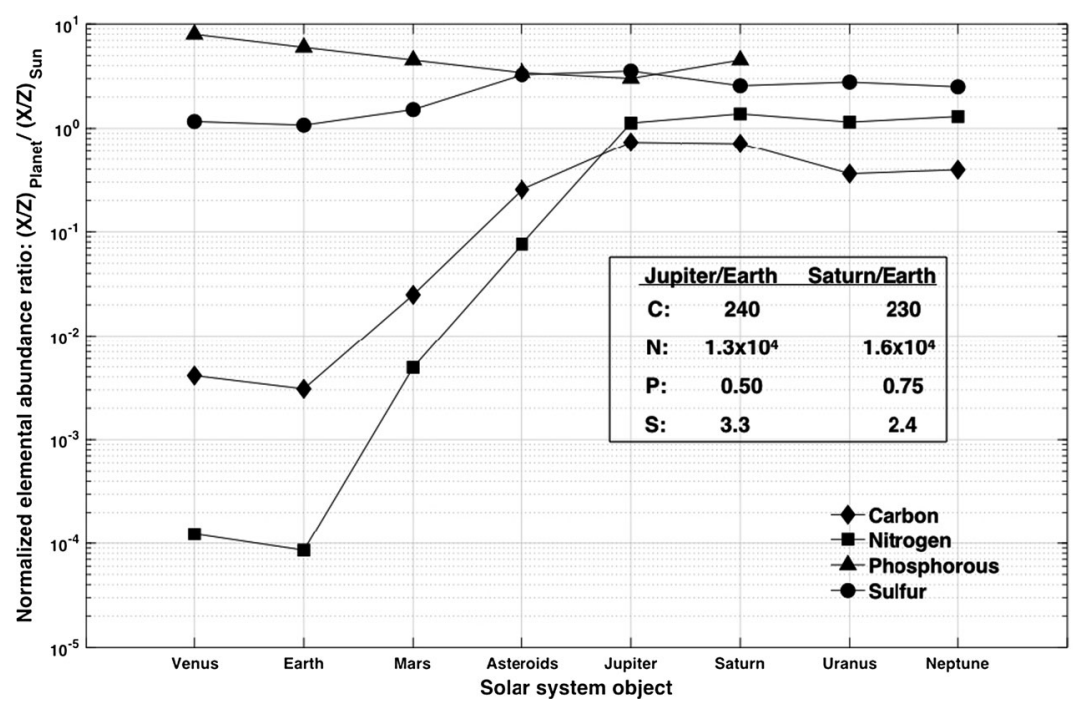

Fig. 2 Elemental abundances across the solar system for the biologically essential light elements carbon, nitrogen, phosphorous, and sulfur. Here we have normalized each value relative to solar abundances. For carbon and nitrogen, and to a lesser extent sulfur, the outer solar system hosts a much larger inventory of these key elements. Depending on the specific conditions of formation and evolution, this plot may be a guide for the relative abundances of key elements within ocean worlds. For additional detail see (Hand et al. 2009; McKay 1991) and references therein

Jet Propulsion Laboratory 2020). As the density of a moon approaches that of water ice, $916.8 \mathrm{~kg} \mathrm{~m}^{-3}$, the prospect of a significant silicate component, and thus water-rock interactions that could yield the heavy elements for life, diminishes. With a decreased inventory of heavier elements, the habitability of lower-density ocean worlds may be compromised. Across the range of candidate ocean worlds considered here, Europa, at $3013 \pm 5 \mathrm{~kg} \mathrm{~m}^{-3}$, is the most dense, while Mimas, at $1150 \pm 4 \mathrm{~kg} \mathrm{~m}^{-3}$, is the least dense (Jet Propulsion Laboratory 2020).

Considering the ocean worlds to have formed primarily from the leaching of bulk chondritic material (Lewis 1971, 1973; Kargel et al. 2000; Sekine et al. 2015), extraction efficiencies of cations such as magnesium, calcium, and potassium - all of which are critical for numerous enzymes (Wackett et al. 2004) - are in the range of $10^{-5}$ to $10^{-6}$ of the initial element mass fraction (Zolotov and Shock 2001). In other words, for one gram in solution, $10^{3}$ kilograms must have been available in the host rock. Extraction factors likely vary considerably over the range of possible formation conditions (Kargel et al. 2000; Neveu et al. 2017), but the basic relationship will persist: the elements in solution are a small fraction of the bulk mass fraction. The availability of biologically essential heavy elements in solution is thus directly related to the total rock fraction of a world, and the extent to which water-rock interactions enable persistent and efficient leaching of those elements.

Europa, Ganymede $\left(1942 \pm 5 \mathrm{~kg} \mathrm{~m}^{-3}\right)$, Callisto $\left(1834 \pm 4 \mathrm{~kg} \mathrm{~m}^{-3}\right)$, and Titan $(1880 \pm 5$ $\mathrm{kg} \mathrm{m}^{-3}$ ) are all sufficiently dense so as to necessitate a significant silicate mass fraction, and, at least in the case of Europa and Ganymede, differentiated iron cores (Anderson et al. 1998; Schubert et al. 2004; Jet Propulsion Laboratory 2020). Spectra of the surfaces of Ganymede and Callisto also reveal potential evidence for endogenous phyllosilicates (McCord et al. 1997, 1998). Titan's surface and atmosphere reveal an abundance of carbon and nitrogen (Hörst 2017; Lebreton et al. 2009; Niemann et al. 2005), and while no direct evidence of 
silicates was detected by Cassini, the density and gravity field of Titan clearly indicate a rocky, silicate component within Titan (Sotin et al. 2009; Iess et al. 2010).

Enceladus emerges as an anomaly. With a density of just $1608 \pm 3 \mathrm{~kg} \mathrm{~m}^{-3}$ it should, perhaps, not be expected to have a strong inventory of heavy elements contained within a rocky interior. Nevertheless, the Cassini data indicates otherwise. Direct detection of salts and silica particles in the plumes and E-ring of Enceladus implicate ongoing water-rock interaction and potentially even seafloor hydrothermal activity (Postberg et al. 2009; Hsu et al. 2015).

Pluto $\left(1890 \pm 60 \mathrm{~kg} \mathrm{~m}^{-3}\right)$ and Triton $\left(2059 \pm 5 \mathrm{~kg} \mathrm{~m}^{-3}\right)$, meanwhile, are denser than Enceladus and are comparable in density to Ganymede, Callisto, and Titan (Jet Propulsion Laboratory 2020). The surface composition of Pluto and Triton has, to date, revealed volatiles and organics on each world (Moore et al. 2016; Grundy et al. 2016, 2010; Cruikshank and Apt 1984). No silicate compounds indicative of rocky interiors have been detected, though the bulk densities indicate otherwise. While the evidence and models for Pluto are still relatively new, it seems clear that Pluto most likely harbors a chemically-rich subsurface region, perhaps even a global ocean. For this reason we place it at the intersection of water and elements in Fig. 1. The evidence for Triton is, unfortunately, limited to the Voyager 2 flyby and telescopic observations (Soderblom et al. 1990; Cruikshank and Apt 1984; Gaeman et al. 2012). Though it may well have the elements and energy needed for life, at this point in time questions still remain about the nature and extent of a putative subsurface ocean. For this reason we position Triton just within the water region of Fig. 1.

An additional consideration for access to heavy elements is that of the nature of the seafloor: Is it made of rock or ice? With large moons of relatively low density, such as Ganymede, Callisto, and Titan, the pressures at the base of their oceans may lead to the formation of higher phases of water ice (McKinnon 1998; Hussmann et al. 2006). As a result, the seafloors of such worlds may be an interface between liquid water and water ice, not liquid water and silicates. Such an interface could limit water-rock interactions and the leaching of elements from silicates.

\subsection{On the Energy Required to Power Life}

The last keystone for habitability is perhaps the hardest to assess via remote sensing with telescopes and flyby spacecraft. The availability of energy - and its availability through time, i.e., power - determines whether or not the chemical pathways in an environment are sufficient to sustain a metabolism capable of genetic repair, growth, and reproduction within an organism. These functions are critical for, and largely define, life as we know it. The energy and power of a system can also modulate the viability of an ecosystem over time, the total biomass in an ecosystem, and the complexity of organisms within that ecosystem.

The most common geochemical metric used to quantify the energy available to do the work of life is that of the negative change in Gibbs free energy. The Gibbs free energy of a given system is the difference between the enthalpy and the entropy at a given temperature. Practically, the enthalpy manifests as a change in heat content, with exothermic reactions yielding a negative change in enthalpy, and endothermic reactions yielding a positive change in enthalpy. The difference between the heat content and the change entropy of the system (at a fixed temperature) is the energy available to do work, which is the Gibbs free energy. As the physical or chemical state of a system changes, a negative change in Gibbs free energy corresponds to the energy available to do work on the system. Biology harnesses this 'free' energy of reactions available in an environment to complete the 'work' of repair, growth, and reproduction. We refer the reader to several books, articles, and reviews on the topic 
for more detail (Gibbs 1948; Nealson 1997; McCollom and Shock 1997; Shock and Boyd 2015).

The energetic limits for life are important to consider in the context of habitability, as the Gibbs free energy within a given system could limit biological activity. As measured by Hoehler et al. (2001), methanogenic archaea and sulfate-reducing bacteria harness a negative change in Gibbs free energy of order $10 \mathrm{~kJ}$ per mole of reactant (carbon dioxide or sulfate), and qualify as some of the most minimally energetically demanding of organisms. Significantly, methanogens, which are deeply rooted in the 16S rRNA tree of life (Hug et al. 2016), have also been cited as possibly representing one of the first metabolisms (Martin et al. 2008).

The chemical reactions through which non-photosynthetic life on Earth harnesses Gibbs free energy can be broadly described as reductant-oxidant, or redox, reactions. (Photosynthesis is also dependent on redox reactions, but photosystem I and photosystem II use the energy available in visible light photons to achieve an excited state for the otherwise energetically unfavorable reduction of carbon dioxide to sugars, and oxidation of water to oxygen.) Redox reactions involve the transfer of an electron, or electrons, from a reductant to an oxidant. On Earth, the variety of compounds available at the interfaces between gas (atmosphere), liquid (oceans, lakes, and rivers), and solid phases (seafloor and continents) yields a host of energetically-rich reactions for life.

While the ocean worlds of the outer solar system may harbor plenty of liquid water and the elements needed to build life, the habitability of these oceans may be constrained by the energetic needs of life. Photosynthetic pathways may be severely limited by ice cover of even just a few tens to hundreds of meters in thickness (Hand et al. 2009). Redox reactions capable of powering chemosynthetic organisms are thus a critical component of maintaining habitable subsurface environments within ocean worlds.

By merit of the rocky, silicate-rich, tidally-active seafloors predicted to exist within several of the candidate ocean worlds, reductants may be available in abundance. Hydrothermal vents and seafloor spreading centers on Earth provide a persistent source of sulfide, methane, hydrogen, and reduced metals (e.g., ferrous iron and massive sulfides), all of which can be utilized as electron donors by microorganisms. Table 1 provides a comparison of internal heat for several worlds, including the Earth. These values may serve as a useful metric for seafloor activity, and reductant supply within ice-covered ocean worlds.

Tidal dissipation within Europa could range from a purely radiogenic $10 \mathrm{~mW} \mathrm{~m}^{-2}$, to an average flux of $800 \mathrm{~mW} \mathrm{~m}^{-2}$, which is an order of magnitude larger than Earth's seafloor flux. The low end value for Europa is unlikely, given the geologic evidence of a young ice shell, but therein lies part of the uncertainty: it may be that much of the tidal dissipation occurs within the ice shell, not in the mantle and seafloor below. The high end flux value corresponds to models for higher internal mantle temperatures $(>1500 \mathrm{~K})$, which in turn lead to greater tidal dissipation and heat production within the mantle and seafloor (Moore et al. 2009). To first approximation, there is no a priori reason to expect large heterogeneity in the heat flux across Europa's surface and seafloor, but latitudinal variations have been long been considered (Ojakangas and Stevenson 1989).

At Enceladus, the south polar terrain provides a dramatic example of a heat flux heterogeneity. The initiation of, and mechanism for sustaining such a heterogeneity is the focus of many modeling efforts (Choblet et al. 2017; Nimmo et al. 2018, 2007; Meyer and Wisdom 2007). The total power radiating from the south polar terrain is approximately $15.8 \pm 3.1 \mathrm{GW}$ (Howett et al. 2011). Regionally, variations in the heat flux from the seafloor could range from nearly zero to $>5000 \mathrm{~mW} \mathrm{~m}^{-2}$ (Choblet et al. 2017).

With high seafloor heat fluxes comparable to, or even exceeding Earth's seafloor heat flux, it is reasonable to expect that water-rock interaction within these two moons cold lead 
Table 1 Comparison of global internal heat production and average heat fluxes for the Earth, Moon, Io, Europa, and Enceladus. Values for the Earth and Moon are dominated by radiogenic decay and secular cooling, whereas those of Io, Europa, and Enceladus are likely dominated by tidal dissipation. All bulk density values are from (Jet Propulsion Laboratory 2020)

\begin{tabular}{|c|c|c|c|c|}
\hline & $\begin{array}{l}\text { Bulk } \\
\text { density } \\
\left(\mathrm{kg} \mathrm{m}^{-3}\right)\end{array}$ & $\begin{array}{l}\text { Global heat } \\
\text { production } \\
(\mathrm{W})\end{array}$ & $\begin{array}{l}\text { Average } \\
\text { flux } \\
\left(\mathrm{mW} \mathrm{m}^{-2}\right)\end{array}$ & $\begin{array}{l}\text { Comments } \\
\text { and } \\
\text { references }\end{array}$ \\
\hline Earth & $5513.6 \pm 0.3$ & $3.2 \times 10^{13}$ & $60-80$ & $\begin{array}{l}\text { Seafloor only. } \\
\text { Approximately } 34 \% \text { of this } \\
\text { total is concentrated around } \\
\text { hydrothermal vents. (Stein } \\
\text { and Stein 1994) }\end{array}$ \\
\hline Moon & $3344 \pm 5$ & $2.8-4.1 \times 10^{11}$ & $9-13$ & $\begin{array}{l}\text { Based on Apollo } 15 \text { and } 17 \\
\text { in situ measurements. } \\
\text { (Siegler and Smrekar 2014) }\end{array}$ \\
\hline Io & $3528 \pm 6$ & $2 \times 10^{14}$ & 2500 & $\begin{array}{l}\text { Fresh lavas are estimated to } \\
\text { reach temperatures of } \\
>1600 \text { K. (Veeder et al. } \\
\text { 1994; } \\
\text { Spencer et al. 2000; } \\
\text { Geissler 2003; } \\
\text { Davies et al. 2001) }\end{array}$ \\
\hline Europa & $3013 \pm 5$ & $10^{11}-10^{13}$ & $10-800$ & $\begin{array}{l}\text { Modeled range includes } \\
\text { radiogenic only, to full tidal } \\
\text { dissipation in the mantle. } \\
\text { (Moore et al. 2009; } \\
\text { Hussmann et al. 2002) }\end{array}$ \\
\hline Enceladus & $1608 \pm 3$ & $>2 \times 10^{10}$ & 0 to $>5000$ & $\begin{array}{l}\text { Much of the flux is } \\
\text { concentrated beneath the } \\
\text { South Polar terrain. (Choblet } \\
\text { et al. 2017; } \\
\text { Porco et al. 2006; } \\
\text { Spencer et al. 2006) }\end{array}$ \\
\hline
\end{tabular}

to significant fluxes of biologically useful reductants. Modeled estimates of the reductant flux resulting from seafloor activity within Europa range from $10^{9}-10^{10}$ moles per year of hydrogen, methane, and sulfide (McCollom 1999; Vance et al. 2016). At Enceladus, Cassini measurements of molecular hydrogen in the plumes leads to an estimate annual flux in the range of $10^{9}$ moles per year (Waite et al. 2017).

Critically, heat flux is not the only consideration. If tidal dissipation is sufficiently vigorous in the seafloor, then the oceanic crust within these moons could be recycled into the mantle, helping to maintain hot, ultramafic material that can directly drive high temperature hydrothermalism, perhaps similar to that found along axis within the spreading centers of Earth's ocean (Von Damm 1990; Elderfield and Schultz 1996). If, however, the oceanic crust is not recycled with the mantle, hydrothermalism may be limited to low temperature, exothermic reactions, such as serpentinization, to drive vent chemistry (Kelley et al. 2001; Allen and Seyfried 2004). Seafloor activity would then be limited to the fracturing depth within the crust, and the time period over which exothermic reactions with ocean water could persist (Lowell and DuBose 2005; Vance et al. 2007). The low internal pressures of ocean worlds (relative to the Earth) may lead to deep fracturing, and orbital resonances may 
drive these worlds through cycles of high- and low-temperature hydrothermalism (Vance et al. 2016; Choblet et al. 2017; Hussmann and Spohn 2004).

Both Europa and Enceladus may sustain reductant-rich oceans through seafloor cycling, and Cassini seems to have verified this to be the case for Enceladus, where an excess of molecular hydrogen emanates from the plumes (Waite et al. 2017). But the availability of oxidants to couple with reductants could be a limiting factor, at least for Enceladus. On Europa, direct observation and mapping of $\mathrm{O}_{2}, \mathrm{H}_{2} \mathrm{O}_{2}$, and sulfate proves that biologically useful oxidants are produced radiolytically on the surface (Carlson et al. 1999a; Spencer and Calvin 2002; Carlson et al. 1999b; Hand and Brown 2013; Brown and Hand 2013; Trumbo et al. 2019). Convection and subsumption of ice may deliver surface oxidants to the ocean over geologic timescales (Pappalardo and Barr 2004; Kattenhorn and Prockter 2014), providing a source of electron acceptors that could combine with reductants, yielding energetically favorable metabolic reactions. Estimates for Europa's oxidant inventory range from $10^{8}-10^{10}$ moles per year of oxygen and hydrogen peroxide, and approximately an order of magnitude higher for sulfate (Hand et al. 2007; Vance et al. 2016). Though peroxide has been implicated on the surface of Enceladus (Newman et al. 2007), subsequent spectroscopic work indicates that methanol may be a better fit to the data (Hodyss et al. 2009; Loeffler and Baragiola 2009). Radiolytic processing of Enceladus' surface is diminished by approximately an order of magnitude, relative to Europa, and thus there may not be an efficient mechanism for generating new oxidants on Enceladus (Paranicas et al. 2012). Redox chemistry on Enceladus, therefore, might be limited to hydrothermally available oxidants, such as $\mathrm{CO}_{2}$ (Hsu et al. 2015; Waite et al. 2017).

On Titan, the availability of oxidants may also be a limiting factor for habitability and life as we know it. (We emphasize again that the prospect of 'weird life' on Titan, utilizing hydrocarbon liquids as the solvent for life, is beyond the scope of this manuscript.) The surface of Titan is flowing with reductants (Hayes et al. 2008), and though tidal heating is relatively small within Titan $\left(5 \mathrm{~mW} \mathrm{~m}^{-2}\right.$ ) (Nimmo and Bills 2010), a deep subsurface ocean likely exists and may be leaching through a poorly differentiated interior (Nimmo and Bills 2010; Sotin et al. 2009). Such water-rock interactions could yield reductants directly within the ocean, but as with Enceladus the primary oxidant would be carbon dioxide. Photolytic chemistry in the upper atmosphere leads to the production of $\mathrm{CO}$ and $\mathrm{CO}_{2}$ (Hörst et al. 2008; Hörst 2017), both of which could add to the oxidant inventory, but transport of these products to the subsurface ocean seems unlikely.

A final important consideration for oxidant production is the mass, and hence surface pressure, of a moon. Europa (and Ganymede) have significant oxidant production via radiolysis, and they are also massive enough (and cold enough) to retain the radiolytic products as condensed phases. At Enceladus, the temperatures are comparable to Europa and Ganymede, but the low gravity $\left(0.113 \mathrm{~m} \mathrm{~s}^{-2}\right)$ is insufficient to retain volatile oxidants such as $\mathrm{O}_{2}$ (Fray and Schmitt 2009). Coupled with this is the role that Io plays in the Jovian system. Volcanism on Io, and the resulting plasma torus, delivers approximately $10^{8}$ sulfur atoms $\mathrm{cm}^{-2} \mathrm{~s}^{-1}$ to Europa (Johnson et al. 2004). Subsequent radiolytic processing of this relatively heavy, and biologically useful, element leads to the production of sulfates, elemental sulfur, and sulfur dioxide, all of which could help maintain redox reaction in the ocean, if they are transported from the surface ice to the ocean below (Hand et al. 2007; Kattenhorn and Prockter 2014). 


\subsection{Assessing the Astrobiological Potential of Ocean Worlds}

The three keystones for habitability described above provide a framework for assessing the astrobiological potential of the various ocean worlds. Table 2 provides a summary of the evidence - in the form of both observations and well-vetted models - for oceans within moons and worlds of the outer solar system, as well as an assessment of planetary parameters related to the keystones for habitability. As with Fig. 1, we emphasize that this table is meant as a guide for the current state of knowledge from available models and observations; new results and improved models will hopefully refine these assessment in the years and decades to come.

The first column addresses plausibility: do the combination of theory and numerical models of that world support the hypothesis that it could harbor subsurface liquid water? The second column is related to the first, but specifically asks whether or not tidal dissipation within that world could sustain a liquid water ocean. Callisto, for example, experiences essentially no tidal energy dissipation - it is not part of the Laplace resonance that maintains a forced eccentricity - but it has a sufficiently high density, and thus rock mass fraction, so as to sustain an ocean through radiogenic decay. Triton, meanwhile, may have experienced significant internal heating from tides during the early evolution of its orbit around Neptune. In the modern epoch, however, its nearly circular orbit likely imparts very little tidal dissipation.

Column 3 of Table 2 provides one powerful line of evidence for a subsurface ocean. The detection of an induced magnetic field, generated by the interaction of a salt water ocean with a time-varying primary magnetic field, is a very effective way to probe the interior of a world with a magnetometer onboard a spacecraft. During its time in orbit around Jupiter, the Galileo spacecraft flew by Europa, Ganymede, and Callisto, and returned evidence of induced fields around each of the moons. In every case except Callisto, the induced field is best explained by a near-surface, conductive, salty liquid water ocean (Kivelson et al. 1997, 1999, 2002; Khurana et al. 1998). At Callisto, induction may still be indicative of an ocean, but induction in Callisto's ionosphere provides a possible alternative explanation (Hartkorn and Saur 2017).

The Saturnian system does not provide as convenient of a configuration for using induced fields as a signature of subsurface oceans. In contrast with Jupiter, which has a magnetic field that is tilted by 9.6 degrees relative the Jupiter's rotation axis, the tilt of Saturn's magnetic field is nearly zero. The absence of a field tilt at Saturn means that the moons do not experience a strong, short-period, $d B / d t$ capable of driving induced fields within subsurface oceans.

Nevertheless, the Cassini spacecraft and the Huygens probe provided several lines of evidence (obliquity, time variable gravity field, librations) for the presence of a deep ocean in Titan, Enceladus, and possibly even Mimas (Soderlund et al. 2020; Journaux et al. 2020). Before definitive optical detection of the plumes of Enceladus, the plumes were sensed as a magnetic anomaly creating a distortion in the Saturnian field as it draped past Enceladus (Dougherty et al. 2006). This alone is not evidence for an ocean, but it was an important development as the Cassini team worked to understand the dynamics of Enceladus. At Titan, the Huygens probe recorded extremely-low frequency electromagnetic waves in the ionosphere during its descent. The $36 \mathrm{~Hz}$ signal in the atmosphere is best explained by a Schumann-like resonance driven by the corotating plasma of Saturn, and set up between the ionosphere and a conducting subsurface layer within Titan (Béghin et al. 2007). A salty, water-ammonia ocean at a depth of 55-80 km below the surface has emerged as the leading hypothesis for this resonance (Béghin et al. 2012). 


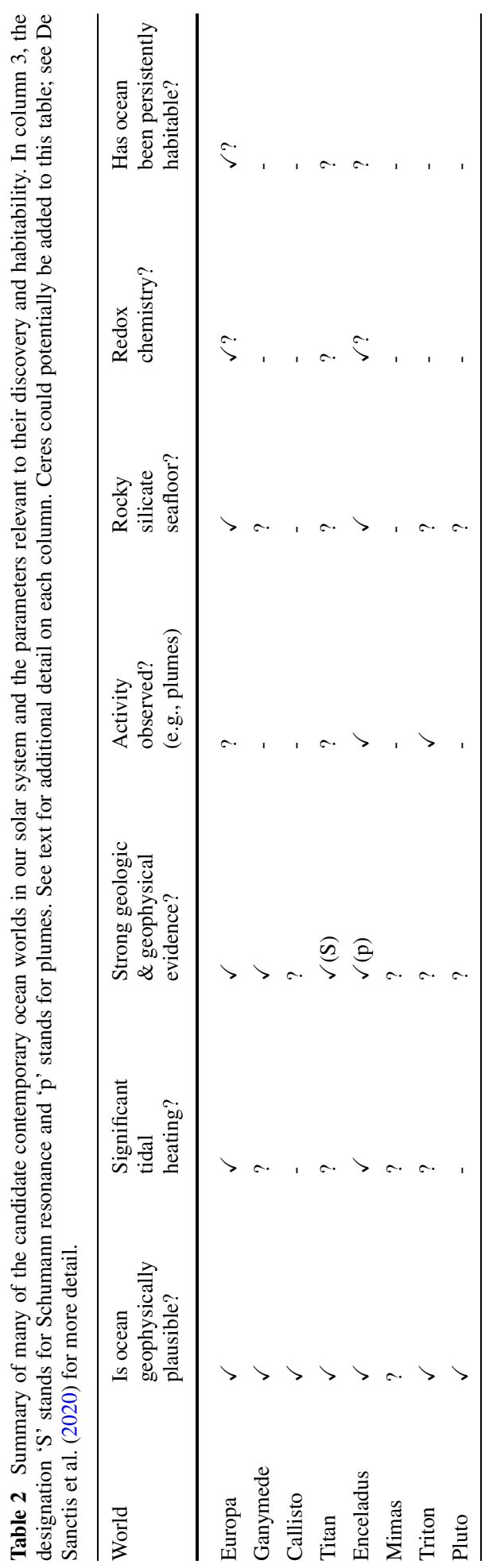


The fourth column of Table 2 indicates on which worlds activity has been observed. This is an important consideration in the context of evidence for, and access to, subsurface oceans. Enceladus and Triton are the only worlds for which definitive evidence exists (Porco et al. 2006; Soderblom et al. 1990). The plumes emanating from Enceladus have been convincingly linked to a salty, subsurface ocean (Postberg et al. 2009), whereas the plumes on Triton could be modulated by seasonal solar irradiance and a solid-state greenhouse effect (Soderblom et al. 1990). Hubble Space Telescope observations have revealed compelling, but still heavily debated, evidence for plume activity on Europa (Sparks et al. 2016, 2017; Roth et al. 2014a, 2014b). In addition, recent analyses of Galileo magnetometer data from the E12 flyby, which was the closest flyby of Europa (206 km at closest approach), also support the presence of plumes erupting from Europa (Jia et al. 2018). Here we place a question mark for activity on Europa, as investigations are ongoing.

The evidence for activity on Titan is also questionable, but compelling. RADAR and near-infrared observations of Titan's surface indicate possible regions of resurfacing, but Titan's thick atmosphere has confounded efforts for definitive evidence (Lopes et al. 2007, 2013; Sotin et al. 2005). Interestingly, the chemistry of the atmosphere, specifically the detection of ${ }^{40} \mathrm{Ar}$ (Waite et al. 2005; Niemann et al. 2005, 2010), also points toward atmospheric exchange with the subsurface.

Columns 5 and 6 relate back to the keystones of the elements and energy needed to build and power life. As detailed above, numerous worlds may have sufficient rock fraction so as to drive water-rock interactions, but to date the evidence and models for Europa and Enceladus provide the strongest indications of silicate seafloors underlying liquid water oceans. In addition, as indicated by Column 6, Europa and Enceladus also emerge as the strongest candidates for sustained redox chemistry. On Europa oxidants are abundant on the surface, but no obvious reductant has yet been directly detected. The internal heat flux ranges detailed above, and the abundance of radiolytically processed iogenic sulfur (Carlson et al. 1999b), indicate that reductants should be available (McCollom 1999; Vance et al. 2016). At Enceladus, reductants have been measured within the plumes (Hsu et al. 2015; Waite et al. 2017) but few oxidants appear readily available. Carbon dioxide, which has been measured in the plumes (Waite et al. 2006), may be the best available oxidant. When combined with molecular hydrogen, carbon dioxide could serve to drive methanogenesis within Enceladus. For both Europa and Enceladus we mark the redox column with a check combined with a question mark. This is meant to indicate that both worlds may have a significant inventory of reductants and oxidants, but in the case of Europa there are not yet measurements of a strong reductant, and at Enceladus there is not yet a measurement of a strong biologically useful oxidant. For Titan, the abundance of silicates and the availability of redox couples in its subsurface ocean is compelling, but much less clear, and thus we mark these two entries in the table with question marks.

The final column of Table 2 addresses a very different, but important parameter for habitability. Time, specifically the persistence of liquid water within these ocean worlds, is essential for the discovery of extant life, and possibly for the origin of life itself. The amount of time needed for life to arise is poorly constrained, but estimates based on laboratory experiments and Earth's rock record range from $<1 \times 10^{5}$ to $3 \times 10^{8}$ years (Orgel 1998; Lazcano and Miller 1996; Sutherland and Opinion 2017; Chyba and Hand 2005).

Of the ocean worlds that may provide the concatenation of conditions needed for habitability, Europa may be the only one for which we can, at this point, claim persistent habitability over the timescale of solar system history (Canup and Ward 2002; Hussmann and Spohn 2004). Enceladus may fall into this category but our understanding of its history and evolution is still a topic of significant debate (Ćuk et al. 2016; 
Zhang et al. 2017; Neveu and Rhoden 2019). Related to this parameter, modeling of tidal heating indicates that in the absence of a liquid layer, the amount of tidal dissipation within Enceladus is about two orders of magnitude lower than present estimates (Tobie et al. 2005). In other words, if the ocean freezes, tidal heating becomes negligible and it may be difficult, or even impossible, for a frozen ocean to become liquid again, even if new orbital resonances provide the potential for increased tidal dissipation. Additional modeling work is needed to more fully understand tidal dissipation through time on small ocean worlds like Enceladus and Mimas.

Based on the concatenation of parameters in Table 2, Jupiter's moon Europa and Saturn's moons Enceladus and Titan have emerged as prime targets in the search for extant life that utilizes water as its solvent and carbon as its primary structural element. (Note, here we are referring to Titan's likely subsurface ocean, not the hydrocarbon lakes and seas that populate the surface.) Much of what we now know about these worlds indicates that they likely have the liquid water, elemental inventory, and possibly even the chemical energy needed to support life. Furthermore, the pressure, temperature, and chemistry predicted for these putative oceans does not preclude life as we know it. As a result, these worlds have become high priority targets for solar system exploration (National Research Council 2012b). In the next section we examine the scientific and technological pathways for future exploration.

\section{Conclusion and Pathways for the Systematic Exploration of Ocean Worlds}

The combination of the Voyager (I and II), Galileo, and Cassini missions precipitated the discovery of oceans beneath the icy shells of moons in the outer solar system. Advancing our understanding of the geophysical, oceanographic, and astrobiological nature of these worlds will require a systematic approach to exploration.

Similar to the strategy for the exploration of Mars, as guided through the Mars program (Shirley and McCleese 1996; McCleese et al. 2001; Hubbard et al. 2002), the exploration of our solar system's ocean worlds will require a well-coordinated strategy that couples civilization-scale science with technological advances that enable a progression of mission capabilities.

Scientifically, ocean worlds are arguably the best place to search for extant life, and a second, independent origin of life. The search for life beyond Earth is one of the longstanding goals of NASA, ESA, other space agencies and new space actors, and the scientific community more broadly (National Research Council 2003, 2012b, 1999, 2002; NASA 2020). For this reason it serves as a strong unifying scientific goal around which to frame the technological development and mission progression.

Furthermore, even in the absence of any signs of life, the exploration of the ocean worlds will open new windows into comparative oceanography, enabling new discoveries and insights regardless of habitability (Hand and German 2018). Oceans will be better understood as a planetary processes if we can study the physical, chemical, and geological conditions across many different oceans in our solar system. This aspect of ocean world exploration is particularly germane to one of the priority science questions detailed in the National Academy's 2015-2025 Sea Change: Decadal Survey of Ocean Sciences: 'What is the geophysical, chemical, and biological character of the subseafloor environment and how does it affect global elemental cycles and understanding of the origin and evolution of life?' (Council et al. 2015). As we develop the tools for exploring oceans beyond Earth, we can also employ these tools to pursue answers to key questions in Earth's ocean and cryosphere. 
The Mars Exploration Program
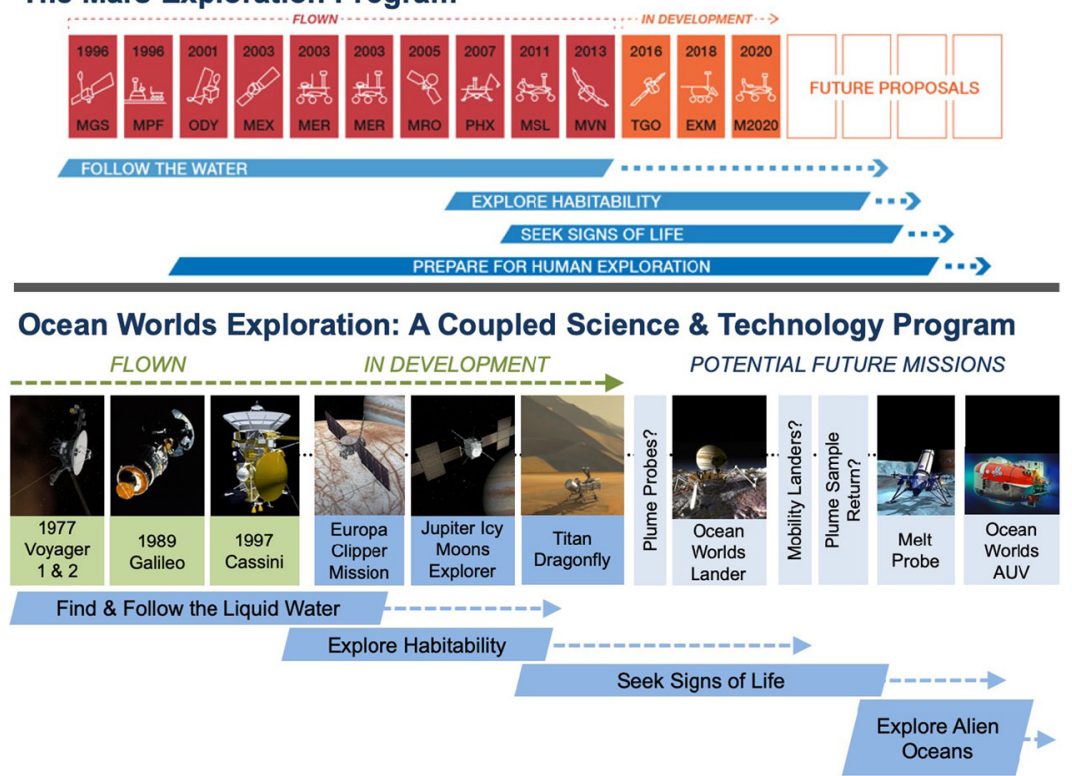

Fig. 3 The Mars Exploration Program brought together a strongly coupled science and technology development program, motivated by the goals of sample return, and preparing for human exploration. The path forward for the systematic exploration of ocean worlds could also benefit greatly from such a coupling. The technologies and mission architectures developed for ocean worlds could be used for multiple targets across decades of exploration. (Mars Exploration image from: http://mars.nasa.gov/programmissions/overview/)

The technological progression for ocean worlds mirrors the same initial steps taken in the Mars program: flyby, orbit, land. Figure 3 provides a diagram from the Mars exploration program, showing the coupled science and technology goals. Also shown is our diagram of how a similar approach might work for exploring the ocean worlds of our solar system. The Voyager, Galileo, and Cassini flybys of the moons of the outer solar system serendipitously accomplished the first key step toward finding signs of life: they found very compelling evidence for vast bodies of subsurface liquid water, a key requirement for life.

Subsequent flyby and orbiting missions such as NASA's Europa Clipper and ESA's Jupiter Icy Moons Explorer (JUICE), which will orbit Ganymede after several flybys of Callisto and Europa, will further our understanding of the ocean worlds of Jupiter, and help assess and constrain their habitability. After those missions, there exists a diversity of mission options, some lower-cost, such as Titan landers and Enceladus plume fly-through missions, and some larger cost, such a Europa lander, and eventually ocean world melt-probes. In 2019, NASA selected Dragonfly, a Titan rotorcraft lander, to be its next New Frontiers class mission. This is a great example of pioneering science on an ocean world for a relatively modest cost.

Despite the diversity of targets and architectures, there is a convergence of technological developments that will aid such exploration. Premier among these are launch vehicles, landing systems, communications, autonomy, and instrumentation. Landing systems with terrain relative navigation to touchdown in a small landing ellipse on icy, airless bodies, can be utilized on any of the ocean worlds (though Titan, of course, offers the benefit of a thick atmosphere in which parachutes can be used). Direct-to-Earth communications has been developed for the Europa Lander mission concept (Chahat et al. 2018), and will also 


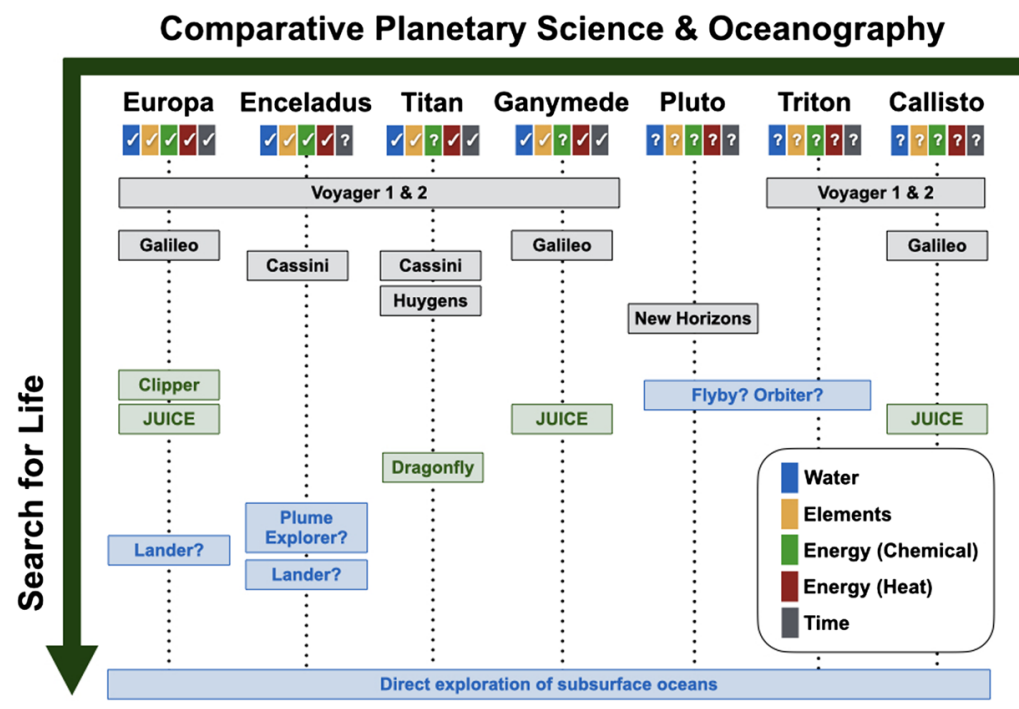

Fig. 4 The past, present, and possible future exploration of candidate ocean worlds. Each world is shown with color bars, and checks and question marks, indicating our assessment of the current state of knowledge for each parameter. In grey are shown the past missions that have provided the data yielding our current understanding. In green are missions that have been selected or approved for development by NASA (Clipper and Dragonfly), and ESA (JUICE). In blue are mission concepts under development that could significantly advance the direct search for signs of life

be used on Dragonfly (Lorenz et al. 2018). Autonomy must become integrated into future missions so as to maximize science return (e.g., efficient sampling), and to reduce risk on missions operating at a distance of many astronomical units from the Earth. Finally, much of the instrumentation needed for investigating ocean worlds and searching for biosignatures has broad applicability across the targets. Instruments such as mass spectrometers, visible and infrared spectrometers, microscopes, and seismometers will be desirable on many future missions. The development of these capabilities is part of the technological and mission progression for ocean world exploration. With these developments, increasingly challenging science questions can be addressed.

To that end, Fig. 4 shows how the study of these candidate ocean worlds has lead to a new field of comparative planetology that includes oceanography. With a rigorous program of exploration, oceans could be studied as a planetary process, while simultaneously advancing the search for life beyond Earth. Across the top are listed the candidate ocean worlds, underneath which are placed color keys for five parameters for assessing habitability. Here we have added heat energy and time, i.e., persistence of a putative ocean, to the three keystones detailed in Sect. 2. Checkmarks and question marks are used to indicate a coarse relative scale for our current state of knowledge. Beneath each world are shown the missions, past (in grey), planned (in green), and under study (blue). These missions are shown relative to an axis indicating the progression toward the capability to search for signs of life. The grand goal in every case, both for astrobiology and for comparative oceanography, would be to someday access the ocean beneath the ice. Creating that capability will first require science and engineering results from orbiting and landed spacecraft that help inform the next steps in exploration.

Following on the flyby and orbiter missions, it is useful to consider the range of possible scientific outcomes and how each outcome affects the path forward for future missions. As 
good as the instrument payloads on flyby and orbiter missions may be, it would be very difficult (though certainly not impossible) for such missions to detect and identify a robust, complementary suite of biosignatures. To that end, the flyby and orbiter missions could yield one of four possible outcomes for the habitability of a given ocean world, 1) it is not habitable (uninhabited), 2) it may be habitable, 3) it is habitable, and 4) it is inhabited.

The first outcome is obviously hard to prove without a comprehensive in situ search, and even then it suffers from the challenge of proving the absence of evidence. Interestingly, ocean worlds are potentially less challenging on this topic than Mars. The geology and geochemistry of the martian surface clearly indicates differences between regions of past liquid water flow, and thus enhanced habitability, and those of more recent, primarily igneous, volcanic origin (arguably less habitable, and certainly less likely to preserve biosignatures). On Mars, specific, discrete regions may have been habitable in the past. Within ocean worlds, however, the availability of liquid water is potentially a globally connected phenomenon, and thus any dissolved compounds that are useful for life may also be available globally. The seafloor and ice-water interface could be more chemically-rich environments, but as with Earth's ocean, global circulation might lead to a globally inhabited ocean, albeit with some regions more biologically productive than others. For this reason, determining that an ocean world is not habitable may not require as exhaustive of a search as the diversity of regions on Mars requires. We note that this attribute of ocean world habitability is also part of what makes planetary protection such an important challenge (National Research Council 2012a). Ultimately, a remote sensing flyby or orbiting mission indicating that an ocean world is not habitable would almost certainly require a follow-on in situ lander for more detailed chemical and geological analyses.

The second and third outcomes - that the ocean world may be habitable, or that it is habitable - both logically lead to a follow-on in situ lander that can provide ground truth for the remote observations, and better determine the chemical constraints on habitability (e.g., $\mathrm{pH}$, salinity, redox couples). Such outcomes also merit a search for potential biosignatures, as habitability may lead to inhabitants, either past or present.

The fourth and final possible outcome - determining that the ocean world is not only habitable, but inhabited - would be very hard to reach with a flyby or orbiting spacecraft. The scientific bar for concluding that life has been detected is very high (Hand et al. 2017), and the claim of life detection should, of course, be the 'hypothesis of last resort' (Sagan et al. 1993). Flybys that yield direct sampling of plumes with high resolution mass spectrometers capable of characterizing large ( $>300 \mathrm{~m} / \mathrm{z}$ ) organic compounds could potentially yield sufficient data for life detection. Similarly, very high spatial resolution imagery of the surface, coupled with high spatial and spectral resolution spectroscopy, could produce very compelling evidence for life, if life is very abundant and is exposed on the surface over spatial scales of meters. Even with such compelling evidence, the next logical step in the progression of missions would almost certainly be to land on the surface. The one exception might be the implementation of a plume sample return mission, but the additional challenges of collecting sufficient sample, and getting it back to Earth safely, would have to be addressed.

Significantly, across the range of possible outcomes described above, landing on the surfaces of ocean worlds is most likely the next logical step. Figure 5 presents an example of how the types of missions might proceed, based on the outcomes from previous missions. We use Europa as the example case as NASA has committed to Europa Clipper, which is a multiple flyby mission. The boxes containing $\mathrm{U}, \mathrm{M}, \mathrm{H}$, and I designate the outcomes described above. As mentioned, and as shown in this diagram, for each of these possible outcomes from the Clipper mission, a surface in situ lander is an important follow-on mission to better understand the remote sensing observations. 


\section{Exploration Pathways for Europa \& the Ocean Worlds}

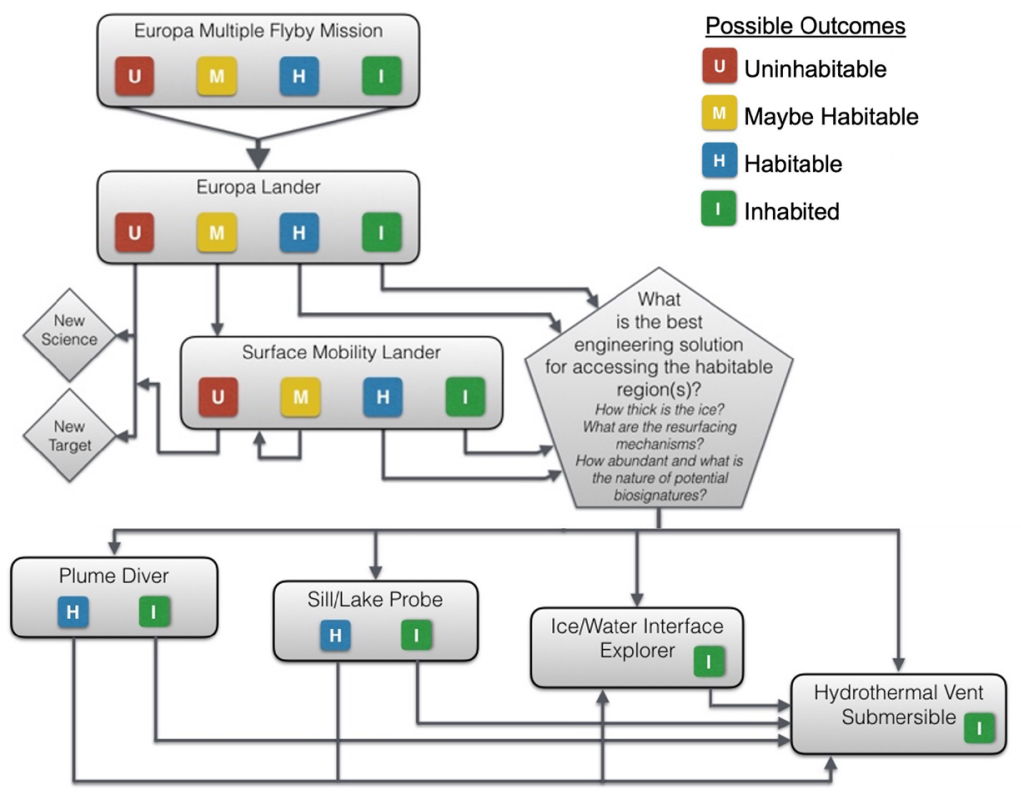

Fig. 5 Pathways for the systematic exploration of Ocean Worlds. Here we show a variety of mission pathways, each of which is contingent on the outcome from the previous mission. Europa is used as the example case, as Europa Clipper (a multiple flyby mission) is already in development. In all cases, the outcome from remote sensing missions leads to in situ sampling with a lander, after which mobility and subsurface access enable further investigations into the habitability, and possible inhabitants, of the ocean. (Adapted from K.P. Hand presentation at the 2017 NASA 'Visions 2050' conference: https://livestream.com/viewnow/Vision2050/videos/150557092)

After the first landed mission, the science outcomes and new engineering information make several pathways possible. If the determination is made that Europa is uninhabitable, then the next step is to pursue other major science questions, and potentially focus on a new ocean world target in the search for signs of life.

If the outcome of the lander is such that Europa may be habitable, but no strong signs of life are detected, then the next step might logically be that of using surface mobility on a subsequent mission to interrogate different sites, at different depths, across Europa. Importantly, the ground truth and engineering information provided by the first lander would provide the information needed to implement mobility on a follow on mission. Surface hardness, regolith depth, and characterization of centimeter- to meter-scale structures not detectable by Clipper will all be useful engineering results from the first lander that will feed forward into future missions (Hand et al. 2017). A similar progression was used on Mars; without the ground truth information from the stationary Viking Landers it would have been very difficult to implement the Mars Pathfinder mission (Muirhead 1997).

Once implemented, surface mobility either results in a determination of the world being uninhabitable, or it becomes recursive on mobility until results lead to an outcome of habitable or inhabited. Stationary or mobile landers could lead to these outcomes. The results from these missions then lead to a critical technical question: What is the best engineering solution for accessing the habitable region(s)? In other words, how do we access the ocean or any subsurface lakes and sills? 
Toward the bottom of Fig. 5 are shown several possible pathways that enable further investigation of the habitable and/or inhabited regions of Europa, and ocean worlds more broadly. Plume divers that target the open crevasses (or chaos regions) from which plumes might emanate, lake and sill probes, robots designed for the ice-water interface, and submersibles capable of reaching seafloor hydrothermal vents all represent specific mission architectures targeting different terrains and regions.

Each of these architectures presents a host of technical challenges that must be addressed over the coming decades if we are ever to directly explore the potentially habitable regions of ocean worlds. Some of these pathways may be easier to implement than others, but all are likely to be useful goals for technical development. For example, the complexity of reaching the depths of Europa's seafloor may not be necessary if life is discovered at the bottom of the ice shell or in liquid water regions within the ice shell. Nevertheless, we will likely someday want to directly study Europa's seafloor as it may hold clues to some of the science questions important to understanding our own ocean, as described above. For example, it may be that if life exists within Europa's ocean, its origin occurred within hydrothermal vent on the seafloor (Baross and Hoffman 1985; Martin et al. 2008; Russell et al. 2017).

The end result of such pathways for ocean world exploration is the technological and mission infrastructure needed to thoroughly explore not just one, but all of the candidate ocean worlds. The coupling of these technological developments to one of humanity's oldest, and yet unanswered of questions, 'Are we alone?', provides a strong and compelling scientific foundation for a well-coordinated program of exploration. Clearly, such exploration and development will take many, many decades, but the investment will open a truly new frontier in space exploration.

Acknowledgements The authors are grateful to, and would like to acknowledge the support of, the International Space Spaces Institute in Bern, Switzerland. KH and CS acknowledge the support of the Jet Propulsion Laboratory, California Institute of Technology, under contract with the National Aeronautics and Space Administration. KH and CS also acknowledge the support of NASA's 'Exploring Ocean Worlds' Research Coordinated Network, managed by the Woods Hole Oceanographic Institution (NASA Award Number: 80NSSC19K1427).

Publisher's Note Springer Nature remains neutral with regard to jurisdictional claims in published maps and institutional affiliations.

\section{References}

D. Allen, W. Seyfried, Serpentinization and heat generation: constraints from Lost City and Rainbow hydrothermal systems. Geochim. Cosmochim. Acta 68(6), 1347-1354 (2004)

E. Ammannito, M. DeSanctis, M. Ciarniello, A. Frigeri, F. Carrozzo, J.P. Combe, B. Ehlmann, S. Marchi, H. McSween, A. Raponi et al., Distribution of phyllosilicates on the surface of Ceres. Science 353(6303), aaf4279 (2016)

J. Anderson, G. Schubert, R. Jacobson, E. Lau, W. Moore, W. Sjorgen, Europa's differentiated internal structure: inferences from four Galileo encounters. Science 281(5385), 2019-2022 (1998)

J. Baross, S. Hoffman, Submarine hydrothermal vents and associated gradient environments as sites for the origin and evolution of life. Orig. Life Evol. Biosph. 15(4), 327-345 (1985)

C. Béghin, F. Simões, V. Krasnoselskikh, K. Schwingenschuh, J. Berthelier, B. Besser, C. Bettanini, R. Grard, M. Hamelin, J. López-Moreno et al., A Schumann-like resonance on Titan driven by Saturn's magnetosphere possibly revealed by the Huygens probe. Icarus 191(1), 251-266 (2007)

C. Béghin, O. Randriamboarison, M. Hamelin, E. Karkoschka, C. Sotin, R.C. Whitten, J.J. Berthelier, R. Grard, F. Simões, Analytic theory of Titan's Schumann resonance: constraints on ionospheric conductivity and buried water ocean. Icarus 218(2), 1028-1042 (2012)

S. Benner, A. Ricardo, M. Carrigan, Is there a common chemical model for life in the universe? Curr. Opin. Chem. Biol. 8(6), 672-689 (2004) 
M. Brown, K. Hand, Salts and radiation products on the surface of Europa. Astron. J. 145(4), 110 (2013)

R.M. Canup, W.R. Ward, Formation of the galilean satellites: conditions of accretion. Astron. J. 124(6), 3404 (2002)

R. Carlson, M. Anderson, R. Johnson, W. Smythe, A. Hendrix, C. Barth, L. Soderblom, G. Hansen, T. McCord, J. Dalton et al., Hydrogen peroxide on the surface of Europa. Science 283(5410), 2062-2064 (1999a)

R. Carlson, R. Johnson, M. Anderson, Sulfuric acid on Europa and the radiolytic sulfur cycle. Science 286(5437), 97-99 (1999b)

N. Chahat, B. Cook, H. Lim, P. Estabrook, All-metal dual-frequency RHCP high-gain antenna for a potential Europa lander. IEEE Trans. Antennas Propag. 66(12), 6791-6798 (2018)

G. Choblet, G. Tobie, C. Sotin, M. Běhounková, O. Čadek, F. Postberg, O. Souček, Powering prolonged hydrothermal activity inside Enceladus. Nat. Astron. 1(12), 841-847 (2017)

C. Chyba, K. Hand, Astrobiology: the study of the living universe. Annu. Rev. Astron. Astrophys. 43, 31-74 (2005)

J.P. Combe, T.B. McCord, F. Tosi, E. Ammannito, F.G. Carrozzo, M.C. De Sanctis, A. Raponi, S. Byrne, M.E. Landis, K.H. Hughson et al., Detection of local $\mathrm{H} 2 \mathrm{O}$ exposed at the surface of Ceres. Science 353(6303), aaf3010 (2016)

N.R. Council et al., Sea Change: 2015-2025 Decadal Survey of Ocean Sciences (National Academies Press, Washington, 2015)

A. Coustenis, T. Tokano, M. Burger, T. Cassidy, R. Lopes, R. Lorenz, K. Retherford, G. Schubert, Atmospheric/exospheric characteristics of icy satellites. Space Sci. Rev. 153(1-4), 155-184 (2010)

D.P. Cruikshank, J. Apt, Methane on triton: physical state and distribution. Icarus 58(2), 306-311 (1984)

M. Ćuk, L. Dones, D. Nesvornỳ, Dynamical evidence for a late formation of Saturn's moons. Astrophys. J. 820(2), 97 (2016)

A.G. Davies, L.P. Keszthelyi, D.A. Williams, C.B. Phillips, A.S. McEwen, R.M. Lopes, W.D. Smythe, L.W. Kamp, L.A. Soderblom, R.W. Carlson, Thermal signature, eruption style, and eruption evolution at Pele and Pillan on Io. J. Geophys. Res., Planets 106(E12), 33079-33103 (2001)

M. De Sanctis, E. Ammannito, H. McSween, A. Raponi, S. Marchi, F. Capaccioni, M. Capria, F. Carrozzo, M. Ciarniello, S. Fonte et al., Localized aliphatic organic material on the surface of Ceres. Science 355(6326), 719-722 (2017)

M. De Sanctis, G. Mitri, J. Castillo-Rogez, C. House, S. Marchi, C.A. Raymond, Y. Sekine, Relict Ocean Worlds: Ceres. Space Sci. Rev. 216(4), 1-33 (2020)

M. Dougherty, K. Khurana, F. Neubauer, C. Russell, J. Saur, J. Leisner, M. Burton, Identification of a dynamic atmosphere at Enceladus with the Cassini magnetometer. Science 311(5766), 1406-1409 (2006)

H. Elderfield, A. Schultz, Mid-ocean ridge hydrothermal fluxes and the chemical composition of the ocean. Annu. Rev. Earth Planet. Sci. 24(1), 191-224 (1996)

A. Ermakov, R. Fu, J. Castillo-Rogez, C. Raymond, R. Park, F. Preusker, C. Russell, D. Smith, M. Zuber, Constraints on Ceres' internal structure and evolution from its shape and gravity measured by the dawn spacecraft. J. Geophys. Res., Planets 122(11), 2267-2293 (2017)

N. Fray, B. Schmitt, Sublimation of ices of astrophysical interest: a bibliographic review. Planet. Space Sci. 57(14-15), 2053-2080 (2009)

R.R. Fu, A.I. Ermakov, S. Marchi, J.C. Castillo-Rogez, C.A. Raymond, B.H. Hager, M.T. Zuber, S.D. King, M.T. Bland, M.C. De Sanctis et al., The interior structure of Ceres as revealed by surface topography. Earth Planet. Sci. Lett. 476, 153-164 (2017)

J. Gaeman, S. Hier-Majumder, J.H. Roberts, Sustainability of a subsurface ocean within Triton's interior. Icarus 220(2), 339-347 (2012)

P.E. Geissler, Volcanic activity on Io during the Galileo era. Annu. Rev. Earth Planet. Sci. 31(1), 175-211 (2003)

J.W. Gibbs, The Collected Works of J. Willard Gibbs, vol. I (Yale University Press, New Haven, 1948)

W. Grundy, L. Young, J. Stansberry, M. Buie, C. Olkin, E. Young, Near-infrared spectral monitoring of Triton with IRTF/SpeX II: spatial distribution and evolution of ices. Icarus 205(2), 594-604 (2010)

W. Grundy, R. Binzel, B. Buratti, J. Cook, D. Cruikshank, C. Dalle Ore, A. Earle, K. Ennico, C. Howett, A. Lunsford et al., Surface compositions across Pluto and Charon. Science 351(6279), aad9189 (2016)

K.P. Hand, M.E. Brown, Keck II observations of hemispherical differences in H2O2 on Europa. Astrophys. J. Lett. 766(2), L21 (2013)

K.P. Hand, C.R. German, Exploring ocean worlds on Earth and beyond. Nat. Geosci. 11(1), $2-4$ (2018)

K. Hand, R. Carlson, C. Chyba, Energy, chemical disequilibrium, and geological constraints Europa. Astrobiology 7(6), 1006-1022 (2007)

K. Hand, C. Chyba, J. Priscu, R. Carlson, K. Nealson, Astrobiology and the potential for life on Europa, in Europa (University of Arizona Press, Tucson, 2009), pp. 589-629 
K. Hand, A. Murray, J. Garvin, W. Brinckerhoff, B. Christner, K. Edgett, B. Ehlmann, C. German, A. Hayes, T. Hoehler, S. Horst, J. Lunine, K. Nealson, C. Paranicas, B. Schmidt, D. Smith, A. Rhoden, M. Russell, A. Templeton, P. Willis, R. Yingst, C. Phillips, M. Cable, K. Craft, A. Hofmann, T. Nordheim, R. Pappalardo, The Project Engineering Team: Report of the Europa Lander Science Definition Team (National Aeronautics and Space Administration, Washington, 2017)

O. Hartkorn, J. Saur, Induction signals from Callisto's ionosphere and their implications on a possible subsurface ocean. J. Geophys. Res. Space Phys. 122(11), 11-677 (2017)

A. Hayes, O. Aharonson, P. Callahan, C. Elachi, Y. Gim, R. Kirk, K. Lewis, R. Lopes, R. Lorenz, J. Lunine et al., Hydrocarbon lakes on Titan: distribution and interaction with a porous regolith. Geophys. Res. Lett. 35(9), L09204 (2008)

M. Hesse, J. Castillo-Rogez, Thermal evolution of the impact-induced cryomagma chamber beneath Occator Crater on Ceres. Geophys. Res. Lett. 46(3), 1213-1221 (2019)

R. Hodyss, C.D. Parkinson, P.V. Johnson, J.V. Stern, J.D. Goguen, Y.L. Yung, I. Kanik, Methanol on Enceladus. Geophys. Res. Lett. 36(17), L17103 (2009)

T.M. Hoehler, M.J. Alperin, D.B. Albert, C.S. Martens, Apparent minimum free energy requirements for methanogenic archaea and sulfate-reducing bacteria in an anoxic marine sediment. FEMS Microbiol. Ecol. 38(1), 33-41 (2001)

S.M. Hörst, Titan's atmosphere and climate. J. Geophys. Res., Planets 122(3), 432-482 (2017)

S.M. Hörst, V. Vuitton, R.V. Yelle, Origin of oxygen species in Titan's atmosphere. J. Geophys. Res., Planets 113(E10), E10006 (2008)

C. Howett, J. Spencer, J. Pearl, M. Segura, High heat flow from Enceladus' south polar region measured using 10-600 $\mathrm{cm}^{-1}$ Cassini/CIRS data. J. Geophys. Res., Planets 116(E3), E03003 (2011)

H.W. Hsu, F. Postberg, Y. Sekine, T. Shibuya, S. Kempf, M. Horányi, A. Juhász, N. Altobelli, K. Suzuki, Y. Masaki et al., Ongoing hydrothermal activities within Enceladus. Nature 519(7542), 207-210 (2015)

G.S. Hubbard, F.M. Naderi, J.B. Garvin, Following the water, the new program for mars exploration. Acta Astronaut. 51(1-9), 337-350 (2002)

L.A. Hug, B.J. Baker, K. Anantharaman, C.T. Brown, A.J. Probst, C.J. Castelle, C.N. Butterfield, A.W. Hernsdorf, Y. Amano, K. Ise et al., A new view of the tree of life. Nat. Microbiol. 1(5), 16048 (2016)

H. Hussmann, T. Spohn, Thermal-orbital evolution of Io and Europa. Icarus 171(2), 391-410 (2004)

H. Hussmann, T. Spohn, K. Wieczerkowski, Thermal equilibrium states of Europa's ice shell: implications for internal ocean thickness and surface heat flow. Icarus 156(1), 143-151 (2002)

H. Hussmann, F. Sohl, T. Spohn, Subsurface oceans and deep interiors of medium-sized outer planet satellites and large trans-neptunian objects. Icarus 185(1), 258-273 (2006)

L. Iess, N.J. Rappaport, R.A. Jacobson, P. Racioppa, D.J. Stevenson, P. Tortora, J.W. Armstrong, S.W. Asmar, Gravity field, shape, and moment of inertia of Titan. Science 327(5971), 1367-1369 (2010)

L. Iess, R.A. Jacobson, M. Ducci, D.J. Stevenson, J.I. Lunine, J.W. Armstrong, S.W. Asmar, P. Racioppa, N.J. Rappaport, P. Tortora, The tides of Titan. Science 337(6093), 457-459 (2012)

Jet Propulsion Laboratory, C.I.o.T., Solar System Dynamics: Ephemerides, orbits, and physical characteristics (2020). https://ssd.jpl.nasa.gov

X. Jia, M.G. Kivelson, K.K. Khurana, W.S. Kurth, Evidence of a plume on Europa from Galileo magnetic and plasma wave signatures. Nat. Astron. 2(6), 459-464 (2018)

R. Johnson, Energetic Charged-Particle Interactions with Atmospheres and Surfaces (Springer, New York, 1990)

R. Johnson, The magnetospheric plasma-driven evolution of satellite atmospheres. Astrophys. J. 609, L99L102 (2004)

R. Johnson, R. Carlson, J. Cooper, C. Paranicas, M. Moore, M. Wong, Radiation effects on the surfaces of the Galilean satellites, in The Planet, Satellites and Magnetosphere, first edn., ed. by F. Bagenal, T. Dowling, W. McKinnon Jupiter (Cambridge University Press, Cambridge, 2004), pp. 485-512

B. Journaux, K. Kalousova, C. Sotin, T. Tobie, S. Vance, J. Saur, O. Bollengier L. Noack, T. RückriemenBez, T. Van Hoolst, K.M. Soderlund J.M. Brown, Large ocean worlds with high-pressure ices. Space Sci. Rev. (2020). https://doi.org/10.1007/s11214-019-0633-7

J. Kargel, J. Kaye, J. Head, G. Marion, R. Sassen, J. Crowley, O. Ballesteros, S. Grant, D. Hogenboom, Europa's crust and ocean: origin, composition, and the prospects for life. Icarus 148(1), 226-265 (2000)

J. Kasting, D. Whitmire, R. Reynolds, Habitable zones around main sequence stars. Icarus 101(1), 108-128 (1993)

S.A. Kattenhorn, L.M. Prockter, Evidence for subduction in the ice shell of Europa. Nat. Geosci. 7(10), $762-767$ (2014)

P.C. Keenan, R.C. McNeil, The Perkins catalog of revised MK types for the cooler stars. Astrophys. J. Suppl. Ser. 71, 245-266 (1989)

D. Kelley, J. Karson, D. Blackman, G. Früh-Green, D. Butterfield, M. Lilley, E. Olson, M. Schrenk, K. Roe, G. Lebon et al., An off-axis hydrothermal vent field near the Mid-Atlantic Ridge at 30 N. Nature 412(6843), 145-149 (2001) 
K. Khurana, M. Kivelson, D. Stevenson, G. Schubert, C. Russell, R. Walker, C. Polanskey, Induced magnetic fields as evidence for subsurface oceans in Europa and Callisto. Nature 395(6704), 749-751 (1998)

M. Kivelson, K. Khurana, S. Joy, C. Russell, D. Southwood, R. Walker, C. Polanskey, Europa's magnetic signature: report from Galileo's pass on 19 December 1996. Science 276(5316), 1239-1241 (1997)

M. Kivelson, K. Khurana, L. Bennett, S. Joy, C. Russell, R. Walker, C. Zimmer, D. Stevenson, C. Polanskey, Europa and Callisto: induced or intrinsic fields in a periodically varying plasma environment. J. Geophys. Res. 104(A3), 4609-4626 (1999)

M. Kivelson, K. Khurana, C. Russell, M. Volwerk, R. Walker, C. Zimmer, Galileo magnetometer measurements: a stronger case for a subsurface ocean at Europa. Science 289(5483), 1340-1343 (2000)

M. Kivelson, K. Khurana, M. Volwerk, The permanent and inductive magnetic moments of Ganymede. Icarus 157(2), 507-522 (2002)

G. Kuiper, The magnitude of the sun, the stellar temperature scale, and bolometric corrections. Astrophys. J. 88, 429 (1938)

A. Lazcano, K.P. Hand, Frontier or fiction: the last great experiment. Nature 488(7410), 160-161 (2012)

A. Lazcano, S.L. Miller, The origin and early evolution of life: prebiotic chemistry, the pre-RNA world, and time. Cell 85(6), 793-798 (1996)

J.P. Lebreton, A. Coustenis, J. Lunine, F. Raulin, T. Owen, D. Strobel, Results from the Huygens probe on Titan. Astron. Astrophys. Rev. 17(2), 149-179 (2009)

J.S. Lewis, Satellites of the outer planets: their physical and chemical nature. Icarus 15(2), 174-185 (1971)

J.S. Lewis, Chemistry of the planets. Annu. Rev. Phys. Chem. 24(1), 339-351 (1973)

M. Loeffler, R. Baragiola, Is the $3.5 \mu \mathrm{m}$ infrared feature on Enceladus due to hydrogen peroxide? Astrophys. J. Lett. 694(1), L92 (2009)

R.M. Lopes, K. Mitchell, E.R. Stofan, J.I. Lunine, R. Lorenz, F. Paganelli, R.L. Kirk, C. Wood, S.D. Wall, L. Robshaw et al., Cryovolcanic features on Titan's surface as revealed by the Cassini Titan Radar Mapper. Icarus 186(2), 395-412 (2007)

R.M. Lopes, R.L. Kirk, K.L. Mitchell, A. LeGall, J.W. Barnes, A. Hayes, J. Kargel, L. Wye, J. Radebaugh, E. Stofan et al., Cryovolcanism on Titan: new results from Cassini radar and vims. J. Geophys. Res., Planets 118(3), 416-435 (2013)

R.D. Lorenz, E.P. Turtle, J.W. Barnes, M.G. Trainer, D.S. Adams, K.E. Hibbard, C.Z. Sheldon, K. Zacny, P.N. Peplowski, D.J. Lawrence et al., Dragonfly: a rotorcraft lander concept for scientific exploration at Titan. Johns Hopkins APL Tech. Dig. 34(3), 14 (2018)

R. Lowell, M. DuBose, Hydrothermal systems on Europa. Geophys. Res. Lett. 32(5), 5202 (2005)

W. Martin, J. Baross, D. Kelley, M.J. Russell, Hydrothermal vents and the origin of life. Nat. Rev. Microbiol. 6(11), 805-814 (2008)

D. McCleese, R. Greeley, G. MacPherson, Science planning for exploring mars. JPL Publ. 1, 1-47 (2001)

T. McCollom, Methanogenesis as a potential source of chemical energy for primary biomass production by autotrophic organisms in hydrothermal systems on Europa. J. Geophys. Res. 104(E12), 30729-30742 (1999)

T. McCollom, E. Shock, Geochemical constraints on chemolithoautotrophic metabolism by microorganisms in seafloor hydrothermal systems. Geochim. Cosmochim. Acta 61(20), 4375-4391 (1997)

T. McCord, R. Carlson, W. Smythe, G. Hansen, R. Clark, C. Hibbitts, F. Fanale, J. Granahan, M. Segura, D. Matson et al., Organics and other molecules in the surfaces of Callisto and Ganymede. Science 278(5336), 271-275 (1997)

T. McCord, G. Hansen, P. Martin, C. Hibbitts, Non-water-ice constituents in the surface material of the icy Galilean satellites from the Galileo near-infrared mapping spectrometer investigation. J. Geophys. Res. 103(E4), 8603-8626 (1998)

C.P. McKay, Urey prize lecture: planetary evolution and the origin of life. Icarus 91(1), 93-100 (1991)

W.B. McKinnon, Geodynamics of icy satellites, in Solar System Ices (Springer, Dordrecht, 1998), pp. 525550

J. Meyer, J. Wisdom, Tidal heating in Enceladus. Icarus 188(2), 535-539 (2007)

W.B. Moore, H. Hussmann, R. Pappalardo, W. McKinnon, K. Khurana, Thermal evolution of Europa's silicate interior, in Europa (University of Arizona Press, Tucson, 2009), p. 369

J.M. Moore, W.B. McKinnon, J.R. Spencer, A.D. Howard, P.M. Schenk, R.A. Beyer, F. Nimmo, K.N. Singer, O.M. Umurhan, O.L. White et al., The geology of Pluto and Charon through the eyes of new horizons. Science 351(6279), 1284-1293 (2016)

B.K. Muirhead, Mars pathfinder flight system integration and test, in IEEE Aerospace Conference, vol. 4 (IEEE, Piscataway, 1997), pp. 191-205

NASA, Explore: Science 2020-2024, a vision for science excellence. Tech. Rep. (NASA Science Mission Directorate, 2020)

National Research Council, A Science Strategy for the Exploration of Europa (National Academies Press, Washington, 1999) 
National Research Council, Signs of Life: A Report Based on the April 2000 Workshop on Life Detection Techniques (National Academies Press, Washington, 2002)

National Research Council, New Frontiers in the Solar System an Integrated Exploration Strategy (National Academies Press, Washington, 2003)

National Research Council, Assessment of Planetary Protection Requirements for Spacecraft Missions to Icy Solar System Bodies (National Academies Press, Washington, 2012a)

National Research Council, Vision and Voyages for Planetary Science in the Decade 2013-2022 (National Academies Press, Washington, 2012b)

K. Nealson, The limits of life on Earth and searching for life on Mars. J. Geophys. Res. 102(E10), 23-675 (1997)

M. Neveu, S.J. Desch, Geochemistry, thermal evolution, and cryovolcanism on Ceres with a muddy ice mantle. Geophys. Res. Lett. 42(23), 10-197 (2015)

M. Neveu, A.R. Rhoden, Evolution of Saturn's mid-sized moons. Nat. Astron. 3(6), 543-552 (2019)

M. Neveu, S.J. Desch, J.C. Castillo-Rogez, Aqueous geochemistry in icy world interiors: equilibrium fluid, rock, and gas compositions, and fate of antifreezes and radionuclides. Geochim. Cosmochim. Acta 212, 324-371 (2017)

S. Newman, B. Buratti, R. Jaumann, J. Bauer, T. Momary, Hydrogen peroxide on Enceladus. Astrophys. J. Lett. 670(2), L143 (2007)

H. Niemann, S. Atreya, S. Bauer, G. Carignan, J. Demick, R. Frost, D. Gautier, J. Haberman, D. Harpold, D. Hunten et al., The abundances of constituents of Titan's atmosphere from the GCMS instrument on the Huygens probe. Nature 438(7069), 779-784 (2005)

H. Niemann, S. Atreya, J. Demick, D. Gautier, J. Haberman, D. Harpold, W. Kasprzak, J. Lunine, T. Owen, F. Raulin, Composition of Titan's lower atmosphere and simple surface volatiles as measured by the Cassini-Huygens probe gas chromatograph mass spectrometer experiment. J. Geophys. Res., Planets 115(E12), E12006 (2010)

F. Nimmo, B. Bills, Shell thickness variations and the long-wavelength topography of Titan. Icarus 208(2), 896-904 (2010)

F. Nimmo, J. Spencer, R. Pappalardo, M. Mullen, Shear heating as the origin of the plumes and heat flux on Enceladus. Nature 447(7142), 289 (2007)

F. Nimmo, D. Hamilton, W. McKinnon, P. Schenk, R. Binzel, C. Bierson, R. Beyer, J. Moore, S. Stern, H. Weaver et al., Reorientation of sputnik Planitia implies a subsurface ocean on Pluto. Nature 540(7631), 94-96 (2016)

F. Nimmo, A.C. Barr, M. Behounková, W.B. McKinnon, The thermal and orbital evolution of Enceladus: observational constraints and models, in Enceladus and the Icy Moons of Saturn, vol. 475 (2018), pp. 7994

G. Ojakangas, D. Stevenson, Thermal state of an ice shell on Europa. Icarus 81(1), 220-241 (1989)

L.E. Orgel, The origin of life-how long did it take? Orig. Life Evol. Biosph. 28(1), 91-96 (1998)

R. Pappalardo, A. Barr, The origin of domes on Europa: the role of thermally induced compositional diapirism. Geophys. Res. Lett. 31(1), L01701 (2004)

C. Paranicas, E. Roussos, N. Krupp, P. Kollmann, A. Hendrix, T. Cassidy, R. Johnson, P. Schenk, G. Jones, J. Carbary et al., Energetic charged particle weathering of Saturn's inner satellites. Planet. Space Sci. 61(1), 60-65 (2012)

C. Porco, P. Helfenstein, P. Thomas, A. Ingersoll, J. Wisdom, R. West, G. Neukum, T. Denk, R. Wagner, T. Roatsch et al., Cassini observes the active south pole of Enceladus. Science 311(5766), 1393-1401 (2006)

F. Postberg, S. Kempf, J. Schmidt, N. Brilliantov, A. Beinsen, B. Abel, U. Buck, R. Srama, Sodium salts in E-ring ice grains from an ocean below the surface of Enceladus. Nature 459(7250), 1098-1101 (2009)

L. Roth, K.D. Retherford, J. Saur, D.F. Strobel, P.D. Feldman, M.A. McGrath, F. Nimmo, Orbital apocenter is not a sufficient condition for HST/STIS detection of Europa's water vapor aurora. Proc. Natl. Acad. Sci. 111(48), E5123-E5132 (2014a)

L. Roth, J. Saur, K.D. Retherford, D.F. Strobel, P.D. Feldman, M.A. McGrath, F. Nimmo, Transient water vapor at Europa's south pole. Science 343(6167), 171-174 (2014b)

L. Rothschild, R. Mancinelli, Life in extreme environments. Nature 409, 1092-1101 (2001)

M.J. Russell, A.E. Murray, K.P. Hand, The possible emergence of life and differentiation of a shallow biosphere on irradiated icy worlds: the example of Europa. Astrobiology 17(12), 1265-1273 (2017)

C. Sagan, W. Thompson, R. Carlson, D. Gurnett, C. Hord, A search for life on Earth from the Galileo spacecraft. Nature 365(6448), 715-721 (1993)

E.E. Salpeter, The luminosity function and stellar evolution. Astrophys. J. 121, 161 (1955)

P.M. Schenk, K. Zahnle, On the negligible surface age of triton. Icarus 192(1), 135-149 (2007)

G. Schubert, J. Anderson, T. Spohn, W. McKinnon, Interior Composition, Structure and Dynamics of the Galilean Satellites (Cambridge University Press, Cambridge, 2004), pp. 281-306 
Y. Sekine, T. Shibuya, F. Postberg, H.W. Hsu, K. Suzuki, Y. Masaki, T. Kuwatani, M. Mori, P.K. Hong, M. Yoshizaki et al., High-temperature water-rock interactions and hydrothermal environments in the chondrite-like core of Enceladus. Nat. Commun. 6(1), 1-8 (2015)

D. Shirley, D. McCleese, Mars exploration program strategy-1995-2020, in 34th Aerospace Sciences Meeting and Exhibit (1996), p. 333

E. Shock, E. Boyd, Principles of geobiochemistry. Elements 11(6), 395-401 (2015)

M. Siegler, S. Smrekar, Lunar heat flow: regional prospective of the Apollo landing sites. J. Geophys. Res., Planets 119(1), 47-63 (2014)

L.A. Soderblom, S. Kieffer, T. Becker, R.H. Brown, A. Cook, C. Hansen, T. Johnson, R.L. Kirk, E. Shoemaker, Triton's geyser-like plumes: discovery and basic characterization. Science 250(4979), 410-415 (1990)

K.M. Soderlund K. Kalousova, J.J. Buffo, C.R. Glein, J.C. Goodman, G. Mitri, G.W. Patterson F. Postberg, M. Rovira-Navarro, T. Rückriemen, J. Saur, B.E. Schmidt, C. Sotin, T. Spohn, G. Tobie, T. Van Hoolst, S. Vance, L.L.A. Vermeersen, Ice-ocean exchange processes in the Jovian and Saturnian satellites. Space Sci. Rev. (2020). https://doi.org/10.1007/s11214-020-00706-6

C. Sotin, R. Jaumann, B. Buratti, R.H. Brown, R. Clark, L. Soderblom, K. Baines, G. Bellucci, J.P. Bibring, F. Capaccioni et al., Release of volatiles from a possible cryovolcano from near-infrared imaging of Titan. Nature 435(7043), 786-789 (2005)

C. Sotin, G. Mitri, N. Rappaport, G. Schubert, D. Stevenson, Titan's interior structure, in Titan from CassiniHuygens (Springer, Dordrecht, 2009), pp. 61-73

W.B. Sparks, K. Hand, M. McGrath, E. Bergeron, M. Cracraft, S. Deustua, Probing for evidence of plumes on Europa with HST/STIS. Astrophys. J. 829(2), 121 (2016)

W.B. Sparks, B.E. Schmidt, M.A. McGrath, K.P. Hand, J. Spencer, M. Cracraft, S.E. Deustua, Active cryovolcanism on Europa? Astrophys. J. Lett. 839(2), L18 (2017)

J. Spencer, W. Calvin, Condensed $\mathrm{O}_{2}$ on Europa and Callisto. Astron. J. 124, 3400-3403 (2002)

J.R. Spencer, K.L. Jessup, M.A. McGrath, G.E. Ballester, R. Yelle, Discovery of gaseous S2 in Io's Pele plume. Science 288(5469), 1208-1210 (2000)

J. Spencer, J. Pearl, M. Segura, F. Flasar, A. Mamoutkine, P. Romani, B. Buratti, A. Hendrix, L. Spilker, R. Lopes, Cassini encounters Enceladus: background and the discovery of a south polar hot spot. Science 311(5766), 1401-1405 (2006)

C. Stein, S. Stein, Constraints on hydrothermal heat flux through the oceanic lithosphere from global heat flow. J. Geophys. Res. 99, 3081-3095 (1994)

S.A. Stern, W.B. McKinnon, Triton's surface age and impactor population revisited in light of kuiper belt fluxes: evidence for small Kuiper belt objects and recent geological activity. Astron. J. 119(2), 945 (2000)

S.A. Stern, W.M. Grundy, W.B. McKinnon, H.A. Weaver, L.A. Young, The Pluto system after new horizons. Annu. Rev. Astron. Astrophys. 56, 357-392 (2018)

J.D. Sutherland, Opinion: studies on the origin of life-the end of the beginning. Nat. Rev. Chem. 1(2), 1-7 (2017)

R. Tajeddine, N. Rambaux, V. Lainey, S. Charnoz, A. Richard, A. Rivoldini, B. Noyelles, Constraints on Mimas' interior from Cassini ISS libration measurements. Science 346(6207), 322-324 (2014)

G. Tobie, A. Mocquet, C. Sotin, Tidal dissipation within large icy satellites: applications to Europa and Titan. Icarus 177(2), 534-549 (2005)

S.K. Trumbo, M.E. Brown, K.P. Hand, H2O2 within chaos terrain on Europa's leading hemisphere. Astron. J. 158(3), 127 (2019)

S. Vance, J. Harnmeijer, J. Kimura, H. Hussmann, B. deMartin, J. Brown, Hydrothermal systems in small ocean planets. Astrobiology 7(6), 987-1005 (2007)

S. Vance, K. Hand, R. Pappalardo, Geophysical controls of chemical disequilibria in Europa. Geophys. Res. Lett. 43(10), 4871-4879 (2016)

G.J. Veeder, D.L. Matson, T.V. Johnson, D.L. Blaney, J.D. Goguen, Io's heat flow from infrared radiometry: 1983-1993. J. Geophys. Res., Planets 99(E8), 17095-17162 (1994)

K. Von Damm, Seafloor hydrothermal activity: black smoker chemistry and chimneys. Annu. Rev. Earth Planet. Sci. 18(1), 173-204 (1990)

L.P. Wackett, A.G. Dodge, L.B. Ellis, Microbial genomics and the periodic table. Appl. Environ. Microbiol. 70(2), 647-655 (2004)

J.H. Waite, H. Niemann, R.V. Yelle, W.T. Kasprzak, T.E. Cravens, J.G. Luhmann, R.L. McNutt, W.H. Ip, D. Gell, V. De La Haye et al., Ion neutral mass spectrometer results from the first flyby of Titan. Science 308(5724), 982-986 (2005)

J. Waite, M. Combi, W. Ip, T. Cravens, R. McNutt, W. Kasprzak, R. Yelle, J. Luhmann, H. Niemann, D. Gell et al., Cassini ion and neutral mass spectrometer: Enceladus plume composition and structure. Science 311(5766), 1419-1422 (2006) 
J. Waite, W. Lewis, B. Magee, J. Lunine, W. McKinnon, C. Glein, O. Mousis, D. Young, T. Brockwell, J. Westlake et al., Liquid water on Enceladus from observations of ammonia and ${ }^{40} \mathrm{Ar}$ in the plume. Nature 460(7254), 487-490 (2009)

J.H. Waite, C.R. Glein, R.S. Perryman, B.D. Teolis, B.A. Magee, G. Miller, J. Grimes, M.E. Perry, K.E. Miller, A. Bouquet et al., Cassini finds molecular hydrogen in the Enceladus plume: evidence for hydrothermal processes. Science 356(6334), 155-159 (2017)

Z. Zhang, A. Hayes, M. Janssen, P. Nicholson, J. Cuzzi, I. de Pater, D. Dunn, Exposure age of Saturn's A and $\mathrm{B}$ rings, and the Cassini division as suggested by their non-icy material content. Icarus 294, 14-42 (2017)

M. Zolotov, E. Shock, Composition and stability of salts on the surface of Europa and their oceanic origin. J. Geophys. Res. 106, 32815-32827 (2001) 